\title{
EFEITOS NA QUALIDADE DE VIDA DE UM PROGRAMA DE ENVELHECIMENTO SAUDÁVEL E ATIVO DURANTE PANDEMIA DE COVID-19
}

\author{
Heloisa Landim Gomes ${ }^{1 *}$, Elizabeth Landim Gomes Siqueira ${ }^{1}$, Cristiane Brasil Francisco ${ }^{1}$, \\ Polyana Teixeira dos Santos, ${ }^{1}$ Nadyson Clayton Abreu da Silva ${ }^{1}$ Maurício Rocha \\ Calomeni $^{1}$
}

\section{RESUMO}

GOMES, H. L. et al. Efeitos na qualidade de vida de um programa de envelhecimento saudável e ativo durante pandemia de COVID-19. Perspectivas Online: Humanas \& Sociais Aplicadas, v.11, n.30, p.104 -114, 2021.

$\mathrm{O}$ estudo teve como objetivo verificar a efetividade das ações da rede de proteção social criada pela Prefeitura de Campos dos Goytacazes/RJ a partir do perfil da qualidade de vida dos idosos que aderiram a um programa de Envelhecimento Saudável e Ativo desenvolvido durante Isolamento Social imposto pela pandemia de COVID-19. A atividade física é importante para os idosos, basicamente para manter seu nível de bem-estar, saúde mental e independência. $\mathrm{O}$ isolamento social devido à pandemia de COVID-19 tem impactado a qualidade de vida de idosos independentemente do estilo de vida antes do isolamento. Experiências implementadas, principalmente pelos poderes públicos, têm procurado efetivar ações de atenção a idosos, pressupondo contribuição para o desenvolvimento de capacidades e autonomia. Foi utilizado um Survey eletrônico composto por perguntas do questionário de Nahas (2017), além de perguntas sobre aspectos socioeconômicos dos participantes. Os links de acesso ao instrumento da pesquisa foram enviados via aplicativo de mensagens aos participantes do projeto de $60+$ Saúde após 4 meses de isolamento social imposto pela pandemia. A amostra foi formada por 356 idosos que aderiram ao programa online de Envelhecimento Saudável e Ativo que promovia a prática de exercícios físicos periódica. Os resultados mostraram que em todos os domínios observados os idosos participantes do estudo tiveram como tendência central o escore 3, o que indica que todos os aspectos são positivos no estilo de vida, e, portanto, fatores de melhoria da qualidade de vida dos participantes. Assim, a adesão ao programa online de Envelhecimento Saudável e Ativo promoveu um impacto positivo no estilo de vida dos idosos.

$\underline{\text { Palavras-chave: Idosos; coronavírus; atividade-física. }}$ 


\title{
EFFECTS ON THE QUALITY OF LIFE OF AN ACTIVE AND HEALTHY AGING PROGRAM DURING THE COVID-19 PANDEMIC
}

\begin{abstract}
The study aimed to verify the effectiveness of the actions of the social protection network created by the Municipality of Campos dos Goytacazes / RJ from presenting the profile of the quality of life of the elderly who adhered to a Healthy and Active Aging program developed during imposed Social Isolation by the COVID-19 pandemic. Physical Activity is essential to aged people, basically to maintain their welfare, mental health, and independence status. The Social Isolation imposed by the COVID-19 pandemic had been impacted the quality of life of aged people independently of their life-style before isolation. Experiences promoted, mainly by public power, have stimulated actions of attention to aged people, assuming that it contributes to the development of capacity and autonomy.

questions of Nahas (2017) questionnaire, besides questions about the socioeconomic aspects of participants. The link for access to the research instrument was sent through message app for the participants of project $60+$ health after 4 months of social isolation. The sample was formed by 356 aged people that were participating in an online program of active and healthy aging that promoted periodic physical exercise practice. The results showed that in all of the observed domains the research aged people had as central tendency the score 3 , which mean that all aspects are positive in their life-style, and, so, aspects of improvement of participants quality of life. Thus, the accession to the online program of active and healthy aging promoted a positive impact on the aged people'slife-style.
\end{abstract} Was used an electronic survey formed by

Keywords: Seniors; coronavirus; Physical-activity.

\footnotetext{
${ }^{1}$ Institutos Superiores de Ensino do CENSA - ISECENSA - Laboratório de Biociências da Motricidade Humana do ISECENSA - LABIMH/ISECENSA - Rua Salvador Correa, 139, Centro, Campos dos Goytacazes, RJ, CEP: 28035-310, Brasil.

(*) e-mail: landim.hl08@gmail.com

Data de recebimento: 29/01/2021. Aceito para publicação: 13/04/2021. Data da publicação: 14/04/2021
}

Persp. Online: hum \& sociais aplicada., Campos dos Goytacazes, 30 (11)104-113- 2021

seer.perspectivasonline.com.br 


\section{INTRODUÇÃO}

Este artigo parte da premissa de que a orientação das políticas públicas para o envelhecimento pauta-se no conhecimento acadêmico-científico e, também no reconhecimento das experiências concretas dos municípios aonde vem se consolidando ações multidisciplinares de atenção ao idoso.

O envelhecimento populacional não é mais uma característica de países em desenvolvimento. Segundo a Organizações das Nações Unidas (ONU, 2002) entre 1970 e 2025 , é esperado um aumento de $223 \%$ de pessoas na faixa etária de 60 anos, em todo o mundo estima-se que em 2050 haverá dois bilhões de pessoas idosas, com 80\% delas vivendo nos países mais ricos. Entretanto teríamos $20 \%$ deste grupo em outras localidades, como elas o Brasil, que segundo o IBGE (2019), a partir de 2025 a pirâmide começa a se inverter, chegando a 2050 com inversão da pirâmide.

Segundo Nahas (2017) envelhecer é um processo natural da vida, que acontece gradualmente, de forma única e individual, é universal e irreversível, onde as habilidades e a saúde são afetadas e sofrem grande influência dos hábitos de vida que aceleram ou tornam mais lento esse processo. Diante disso, o indivíduo idoso pode ser classificado como sendo do grupo de risco, que é o termo utilizado para determinar aqueles mais suscetíveis a diversas doenças infectocontagiosas, como portadores de doenças crônicas não transmissíveis (DCNTs) e comorbidades.

Tavares et al. (2020) relatam que a idade avançada é um fator que incluí indivíduos no grupo de risco, pois está associada a alterações significativas no sistema imune, e, esse declínio da responsividade do sistema imune diminui a capacidade de produção de anticorpos impactando a capacidade de responder a infeções, e reagir de maneira eficiente no combate a doenças provocadas por vírus. No Brasil DCNTs correspondem a 72\% das causas de morte e atingem fortemente os grupos vulneráveis e camadas pobres da população (MINISTÉRIO DA SAÚDE, 2011).

Diante disto, Okuma (2012) aponta que os fatores de risco das DCNTs são modificáveis, e evidencia o expressivo impacto da atividade física regular na prevenção e no tratamento de doenças crônicas inerentes do envelhecimento como também na manutenção funcional do idoso, e como consequências qualidade de vida e aumento da longevidade. Nos dias atuais a correlação entre qualidade de vida e um estilo de vida fisicamente ativo é clara e interdependente, não só entre idosos, mas em pessoas de todas as idades. Na meia-idade e na velhice os riscos potenciais relacionados a inatividade física se potencializam, podendo levar a perda de muitos anos de vida útil (NAHAS, 2017).

No final dos anos 90 o termo "envelhecimento saudável" precisou ser ampliado por não atender a todos os aspectos inseridos nas dimensões sociais, econômicas, culturais, espirituais e civis dos indivíduos em processo de envelhecimento. Dessa forma, a Organização Mundial da Saúde passou a recomendar o termo "envelhecimento ativo", pois o termo "ativo" faz analogia a estar ativo em todas estas dimensões (Kalache e Kickbusch, 1997 apud OMS, 2005). Nesse sentido, políticas públicas internacionais como o Plano de Ação sobre o Envelhecimento (PIAE), aprovado pelos países membros da Organização das Nações

Persp. Online: hum \& sociais aplicada., Campos dos Goytacazes, 30 (11)104-113- 2021 seer.perspectivasonline.com.br 
Unidas (ONU) tem como objetivo principal propor diretrizes que são prioritárias para promoção o envelhecimento ativo e saudável.

O respectivo plano de ação reconhece o envelhecimento como multifatorial, ou seja, envolve aspectos biopsicossociais tais como saúde mental através da profilaxia dos transtornos mentais, diagnóstico e tratamento precoce de doênças, e, melhoria do atendimento ao idoso por meio da capacitação profissional. Além disso, o plano também prevê a conscientização da população jovem da importância de se chegar à velhice com saúde física e mental, e, a consolidação de uma rede de apoio e cuidado para os idosos que envolve família, voluntários e comunidade. Por fim, ainda merece destaque nesse plano, a realização de pesquisas científicas com intuito de se levantar indicadores confiáveis que devem ser utilizados para formulação das políticas públicas voltadas à população que envelhece.

Para Neri (2011) essas experiências, implementadas principalmente pelos poderes públicos, têm procurado efetivar ações de atenção a idosos, pressupondo contribuição para o desenvolvimento de capacidades e autonomia, fortalecimento das relações no âmbito familiar e da comunidade onde vivem e convivem, ampliação do acesso aos direitos de cidadania e reflexões para um processo de envelhecimento com qualidade de vida. Além disso, existem sólidas evidências de que o estilo de vida individual apresenta elevado impacto sobre a saúde e qualidade de vida. Esse impacto pode ser dimensionado em todas as etapas da vida do homem. Nesse sentido, o estilo de vida adotado, durante a fase de desenvolvimento humano, está intrinsicamente ligado a uma velhice saudável (NAHAS, 2017).

Diante do desafio imposto ao poder municipal da cidade de Campos dos Goytacazes/RJ para promoção de um estilo de vida ativo para população idosa, implementouse o Projeto 60+ Saúde desenvolvido pela superintendência dos Direitos do Idoso, dentro da proposta do envelhecimento saudável e ativo. Trata-se de um projeto realizado nas Casas de Convivência, Fundação Municipal de Esportes e nas Vilas Olímpicas em todo município em parceria com a Fundação Municipal de Esportes (FME) e os Institutos Superiores de Ensino do Censa (ISECENSA), promovendo atendimentos em diversas atividades físicas, com a meta de oportunizar atividades para que o idoso envelheça com saúde, através de um estilo de vida saudável.

Todavia, desde a sua declaração de pandemia emitida pela Organização Mundial de Saúde (OMS) em 30 de janeiro de 2020, e devido ao alto poder de contágio do coronavírus (Sars-CoV-2), causador da doença COVID-19, uma das estratégias adotadas visando conter a disseminação do COVID-19 foi o isolamento social, principalmente de indivíduos inseridos em grupos de risco como idosos e pessoas acometidas com doenças crônicas. Dessa forma, um grande número de idosos inseridos no projeto 60+ que tinham um estilo de vida fisicamente ativo tiveram suas atividades físicas hábituais drasticamente reduzidas, o que poderia produzir impactos negativos tanto em sua saúde física quanto mental.

Assim, ainda dentro da proposta da rede de proteção social inserido no Projeto $60+$ Saúde, foi criado o Canal Envelhecimento Ativo com vídeos com foco na atividade física destinado a pessoas com mais de 60 anos, com atenção na saúde preventiva das pessoas que estão envelhecendo. $\mathrm{O}$ objetivo foi estimular a atividade física, uma iniciativa reconhecida como o melhor remédio para o idoso, proporcionando aulas de ginástica, força resistida, caminhada e alongamento, prescritas por profissionais de educação física com volume e intensidade segura para população idosa, além de outras normas de segurança. Dessa forma, o

Persp. Online: hum \& sociais aplicada., Campos dos Goytacazes, 30 (11)104-113- 2021 seer.perspectivasonline.com.br 
Canal Envelhecimento Ativo nesse momento de pandemia de COVID-19, de isolamento social, tornou-se imprescindível para que o idoso pudesse se manter em atividade física regular, com os exercícios sugeridos através de vídeo-aulas.

Por essa razão, este artigo, a partir de uma pesquisa quantitativa, tem o objetivo de verificar e efetividade das ações da rede de proteção social criada pela Prefeitura de Campos dos Goytacazes/RJ, que segundo o Instituto Brasileiro de Geografia e Estatística (IBGE) possui uma população por volta de 55.041 idosos, implementada pela Secretaria Municipal de Envelhecimento Saudável, por meio do Projeto 60+ Saúde através do seu canal digital Envelhecimento Ativo no Youtube durante um periodo de 4 meses de isolamento social, com ações que estimulam a prática de atividade física.

\section{METODOLOGIA}

A metodologia que segue abaixo foi aprovada pelo Comitê de Ética em Pesquisa com Seres Humanos do ISECENSA com CAAE $n^{\circ}$ 35070820.8.0000.5524. Foi utilizado um Survey eletrônico composto por perguntas do Pentáculo do Bem-proposto por Nahas (2017), além de perguntas sobre aspectos socioeconômicos dos participantes. Os links de acesso ao instrumento da pesquisa foram enviados via aplicativo de mensagens aos participantes do projeto de 60 + Saúde após 4 meses de isolamento social imposto pela pandemia de COVID19.

Esses idosos ao receberem o link de acesso ao instrumento de pesquisa foram orientados responder a cada questão do instrumento levando em consideração seu estilo de vida durante o período de isolamento, e caso tivessem alguma dificuldade no preenchimento online do instrumento que solicitassem ajuda a algum membro da família. Sendo assim, a amostra foi formada por 356 idosos que aderiram ao programa online de Envelhecimento Saudável e Ativo, disponibilizado em aulas periódicas. Foram incluídos todos os idosos inseridos no programa 60+, e excluídos os que nunca utilizaram o canal Envelhecimento Ativo no Youtube para prática de exercícios físicos e que não responderam ao questionário durante o período estipulado para o estudo.

Com a utilização do Pentáculo do Bem-estar, uma ferramenta onde sua aplicação consiste em um questionário do Perfil do Estilo de Vida Individual (PEVI) com 15 perguntas para auto avaliação com escala correspondente a 0 (NUNCA) absolutamente não faz parte do seu estilo de vida, 1 (ÀS VEZES) às vezes corresponde ao comportamento, 2 (QUASE SEMPRE) quase sempre verdadeiro, 3 (SEMPRE) que é a completa realização do comportamento considerado (NAHAS, 2017). Esse instrumento de pesquisa foi convertido em formulário online através da ferramenta gratuita do Google Forms, a qual permita ageração de um link que pode ser compartilhado via aplicativos de mensagens, e-mails e redes sociais.

O instrumento foi enviado via aplicativo de mensagens no qual todos os idosos inseridos no programa 60+ já estavam familiarizados por participarem de diferentes grupos do programa. Juntamente com o link foram enviadas instruções detalhadas de como fazer o prenchimeto do formulário online que foi estruturado para fosse o mais intuitivo possível. Foi

Persp. Online: hum \& sociais aplicada., Campos dos Goytacazes, 30 (11)104-113- 2021 seer.perspectivasonline.com.br 
dado o prazo de 15 dias para que todos os idosos respondessem ao questionário, e após esse tempo os dados foram coletados e analizados.

A análise dos dados foi feita em três etapas. Primeiro foi determinada para cada uma das 15 perguntas do questionário do Perfil do Estilo de Vida Individual o quantitativo de respostas atribuídas a cada um dos escores possíveis ( 0 a 3). Em seguida foi calculada a média aritmética do total atribuído a esses escores em cada uma das questões referentes aos componentes do pentatlo do bem estar avaliados. Esse procedimento permitiu se chegar a um valor único que representa o total atribuído a cada escore possível em cada um dos cinco domínios do pentatlo.

Por fim, calculou-se o valor percentual possível, de cada escore, em cada um dos 5 domínios o que permitiu representar a presença de determinado comportamento relacionado à qualidade de vida, no estilo de vida dos participantes.

\section{RESULTADOS E DISCUSSÃO}

A partir dos resultados obtidos foi possível caracterizar os participantes do estudo. Para isso, serão apresentados a seguir os dados referentes ao sexo, escolaridade, faixa etária e renda familiar respectivamente.

A figura 1 nos mostra que a amostra do estudo foi predominantemente do sexo feminino (71\%) e com faixa etária dos 60 aos 74 anos (78\%). Esses dados vão de encontro com o estudo de Harris et al. (2020) que embasados por outros autores afirmam que as pessoas do sexo feminino têm maior probabilidade de se engajarem em programas supervisionados de exercícios físicos, pois dão mais valor e esse tipo de atividade, e, ainda, que os homens têm maior propensão a considerar o estereótipo de que a ginástica seja uma atividade tipicamente feminina. Além disso, no estudo realizado por esses autores constatou-se com relação à faixa etária, que os idosos mais jovens têm maiores taxas de entrada e permanência em programas de exercícios físicos, dado que também vai de encontro aos produzidos no presente estudo. Por fim, os dados relativos à renda familiar e a escolaridade mostram que o programa de exercícios físicos oferecido pelas casas de convivência possuía uma ampla abrangência com idosos diferentes níveis de escolaridade e renda familiar.

Persp. Online: hum \& sociais aplicada., Campos dos Goytacazes, 30 (11)104-113- 2021 seer.perspectivasonline.com.br 


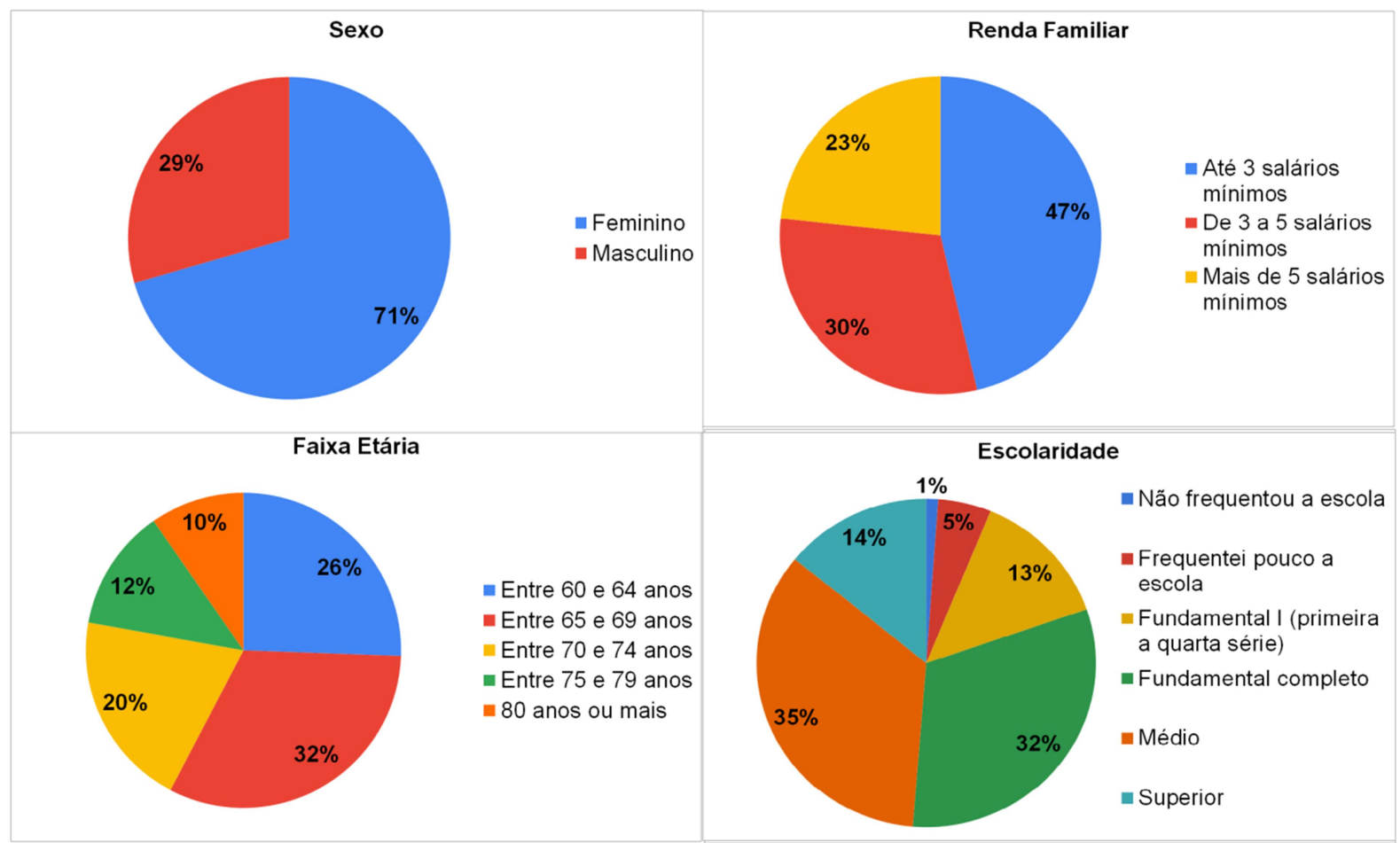

Figura 1. Caracterização dos idosos inseridos no programa 60+ Saúde do município de Campos dos Goytacazes em 2020. Cada gráfico representa o percentual de indivíduos de acordo com os critérios descritos: sexo, faixa etária, renda familiar e escolaridade.

Em seguida são apresentados os gráficos referentes ao perfil do estilo de vida individual dos participantes. Cada gráfico foi produzido a partir do cálculo do valor percentual atribuído a cada uma das quatro opções de respostas dadas para as 14 perguntas que formam o Pentáculo do bem-estar que avaliam aspectos relacionados à qualidade de vida como: alimentação saudável, atividade física, controle do estresse, relacionamentos e comportamento preventivo. 


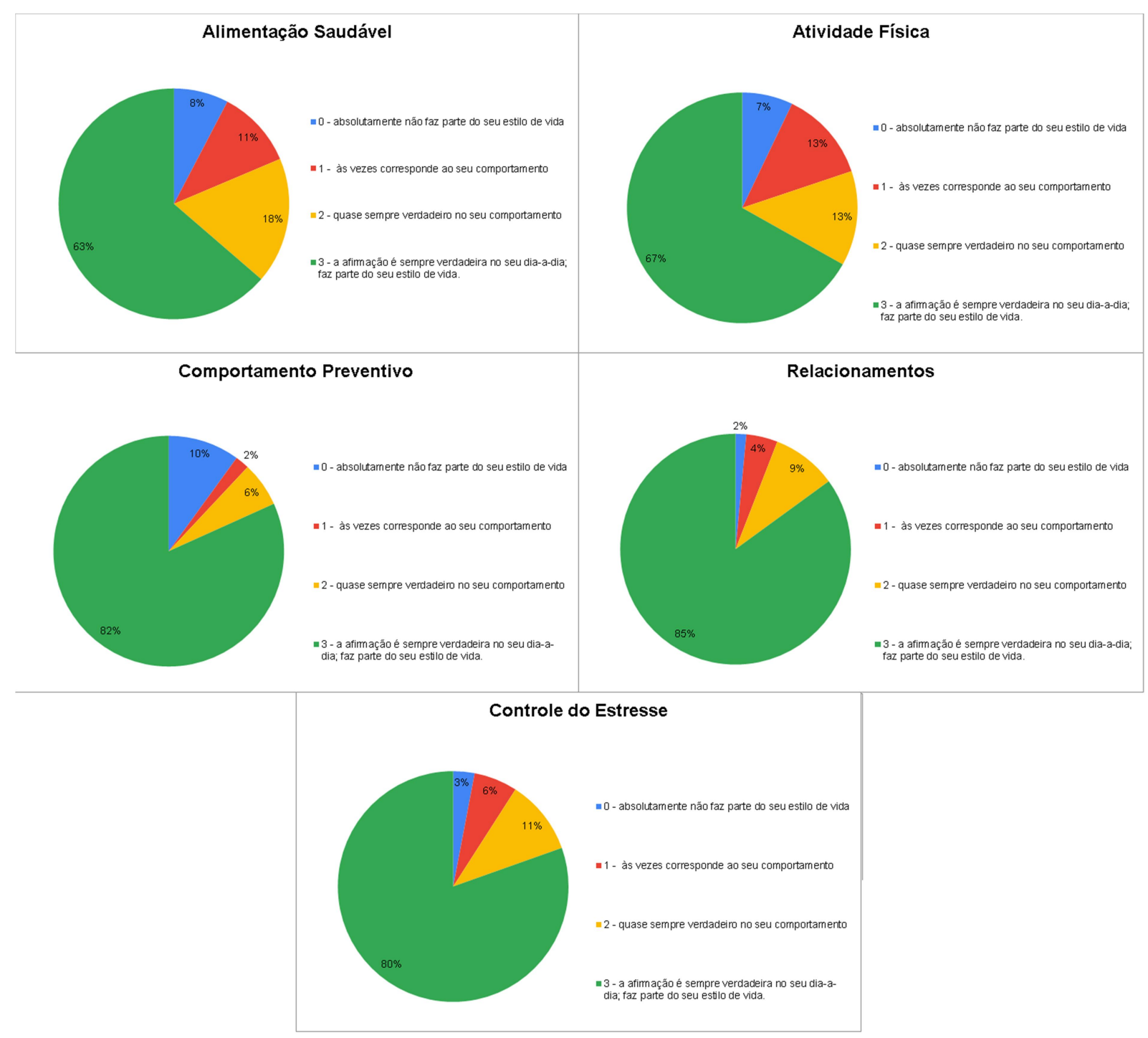

Figura 2. Avaliação dos aspectos relacionados à qualidade de vida de idosos inseridos no programa 60+ Saúde do município de Campos dos Goytacazes em 2020: alimentação saudável, atividade física, controle do estresse, relacionamentos e comportamento preventivo.

Em linhas gerais, a análise das informações contidas na figura 2 aponta que os idosos que aderiram à prática de atividades físicas online, via canal Envelhecimento Ativo, no Youtube, dentro do programa 60+, em sua maioria (75\%) mantiveram, como parte do seu estilo de vida durante os quatro meses de isolamento social observados nesse estudo, hábitos de alimentação saudável, atividade física, comportamento preventivo, relacionamentos e controle do estresse. Em outras palavras, para todas as perguntas que compõem a avaliação do pentáculo do bem estar, a maioria dos idosos escolheram a opção 3 que indica que o hábito relacionado a uma boa qualidade de vida abordado em cada questão está sempre presente no seu dia-a-dia e faz parte do seu estilo de vida.

Em uma visão holística, Nahas (2017) considera qualidade de vida como: "a percepção de bem-estar resultante de um conjunto de parâmetros individuais e socioambientais, modificáveis ou não, que caracterizam as condições em que vive o ser humano". O autor apresenta tais fatores resumidos em dois grupos, Parâmetros 
Socioambientais (moradia, transporte, segurança; assistência médica; condições de trabalho; educação; opções de lazer; meio ambiente; cultura e vida comunitária) e Parâmetros Individuais (hereditariedade; percepção dos parâmetros socioambientais; estilo de vida: hábitos alimentares, controle do estresse, atividade física habitual, relacionamentos e comportamento preventivo).

Interessante destacar que os fatores do pentáculo do bem estar que registraram valores iguais ou acima de $80 \%$ de hábitos associados à melhor qualidade de vida (opção 3 na avaliação do pentatlo do bem estar) foram: comportamento preventivo (82\%), Relacionamentos (85\%), e, Controle do Estresse (80\%). Devido ao isolamento social, esperava-se que os hábitos favoráveis a uma boa qualidade de vida estivessem menos presentes no dia-a-dia desses idosos e, por esta razão, este dado se torna tão importante. Em uma revisão integrativa de literatura feita com 49 trabalhos publicados em diferentes bases de dados, com o objetivo analisar os efeitos do isolamento social imposto pela pandemia de COVID-19 na saúde mental, Pereira el al (2020) encontraram que indivíduos submetidos à isolamento social são mais suscetíveis a desenvolver transtornos mentais, em especial os que estão associados ao estresse, ansiedade e depressão.

Com relação às questões do pentaculo do bem estar relacionadas à alimentação saudável, um número expressivo de idosos afirmou que os hábitos relacionados a esse fator que estão associados a uma boa qualidade de vida estão sempre presentes em sua rotina $(63 \%)$. De fato, tal constatação corrobora com o discutido até este momento onde evidenciouse que $82 \%$ dos participantes do estudo relatam hábitos relacionados a comportamentos preventivos, relacionamento e controle do estresse. Fazzio (2012) afirma, em outra revisão de literatura, que dentre outros fatores, o consumo de alimentos sofre influência da renda, das interações sociais e estado de ânimo dos idosos.

Por fim, o último componente do pentáculo do bem-estar discutido foi a Atividade Física. Nota-se que nesse componente, mais da metade dos idosos (67\%) mantém sempre no seu dia-a-dia, e, consideram como "parte do seu estilo de vida", hábitos relacionados à prática de atividades físicas. Todavia, como discutido na apresentação dos outros fatores do pentáculo do bem-estar, isso também pode indicar que mesmo não tendo hábitos relacionados à prática de atividades físicas presentes no seu dia-a-dia, os hábitos que são mantidos, mesmo que não frequentes, podem contribuir de forma expressiva para adoção de outros hábitos relacionados a melhoria da qualidade de vida. Além disso, para Nahas (2017) os benefícios da prática de atividades físicas durante o envelhecimento dão-se em duas vertentes: individual e na sociedade como um todo. Em relação aos benefícios individuais, estão os aspectos fisiológicos, psicológicos e sociais, sendo que para os idosos, os benefícios considerados de maior relevância são os promovidos em médio e longo prazo, em que ocorrem as modificações estruturais e funcionais.

Considerando a sociedade como um todo, em lugares onde o idoso é mais ativo fisicamente espera-se que o custo com cuidados paliativos, de saúde e atendimento social, sejam reduzidos. Esse mesmo autor também aponta para o fato de que se tratando de uma sociedade que envelhece rápido, como a nossa, o papel do idoso na sociedade tenha que ser redefinido no meio social, valorizando-se a diversa contribuição que ele ainda pode trazer.

\section{CONCLUSÕES}

Persp. Online: hum \& sociais aplicada., Campos dos Goytacazes, 30 (11)104-113- 2021 seer.perspectivasonline.com.br 
A avaliação do pentáculo do bem estar dos participantes do programa $60+$ que se mantiveram fisicamente ativos através do incentivo à prática de exercícios físicos via canal Envelhecimento Ativo no Youtube se mostrou efetiva para manutenção da qualidade de vida dos idosos durante o período de isolamento social. Uma vez que em todos os componentes do pentatlo do bem-estar, a maioria dos participantes declararam ter hábitos relacionados ao bem-estar e qualidade de vida sempre presentes em seu estilo de vida.

Contudo, apesar do número expressivo de participantes incluídos nesse estudo, a falta de um grupo controle e de dados que permitissem comparações entre os escores do bem-estar e qualidade de vida antes do período de isolamento social imposto pela pandemia de COVID19 não permitem que os resultados apresentados possam ser considerados conclusivos.

Todavia, esses mesmos resultados indicam que é viável a implantação de políticas públicas voltadas a promoção de um envelhecimento ativo, e, que estratégias de promoção de exercícios físicos orientados de forma online por profissionais de educação física podem impactar positivamente na qualidade vida de idosos submetidos ao isolamento social.

\section{REFERÊNCIAS}

FAZZIO, D.M.G. Envelhecimento e qualidade de vida-uma abordagem nutricional e alimentar. Revista de divulgação científica Sena Aires, v. 1, n. 1, p. 76-88, 2012.

HARRIS, E.R.A. et al . Motivos da adesão de idosos às Academias da Terceira Idade. Rev. bras. geriatr. gerontol., Rio de Janeiro , v. 23, n. 2, e200117, 2020 . Available from $<$ http://www.scielo.br/scielo.php?script=sci_arttext\&pid=S1809-

98232020000200203\&lng=en\&nrm=iso $>$. access on 27 Jan. 2021. Epub Sep 23, 2020. https://doi.org/10.1590/1981-22562020023.200117.

INSTITUTO BRASILEIRO DE GEOGRAFIA E ESTATÍSTICA - IBGE. Projeção populacional. Rio de Janeiro, 2019. Disponível em: <https://www.ibge.gov.br/ apps/populacao/projecao/>. Acesso em junho de 2020.

MINISTÉRIO DA SAÚDE. Plano de ações estratégicas para o enfrentamento das doenças crônicas não transmissíveis (DCNT) no Brasil 2011-2022. 2011. Disponível em: $<$ https://www.saude.pr.gov.br/sites/default/arquivos_restritos/files/ documento/202004/planonacionalacoesenfret_2012a2015.pdf >. Acesso em novembro de 2020.

NAHAS, M. V. Atividade Física, Saúde e Qualidade de Vida: Conceitos e Sugestões para um Estilo de Vida Ativo. 7. ed. Florianópolis: Ed. do Autor, 2017.

NERI, A. L. Qualidade de vida na velhice: enfoque multidisciplinar. 2. Ed. Campinas: Alínea; 2011.

OKUMA, S. S. O idoso e a atividade Física: Fundamentos e pesquisa. 6 ed. São Paulo: Papirus, 2012.

Persp. Online: hum \& sociais aplicada., Campos dos Goytacazes, 30 (11)104-113- 2021 seer.perspectivasonline.com.br 
UNITED NATIONS POPULATION DIVISION. World population ageing: 1950-2050. New York, 2002.

ORGANIZAÇÃO MUNDIAL DA SAÚDE - OMS. Envelhecimento Ativo: Uma política de Saúde. Brasília: Organização Pan Americana de Saúde, 2005. Disponível em: $<\mathrm{http}$ ///prattein.com.br/home/images/stories/Envelhecimento/ envelhecimento_ativo.pdf $>$. Acesso em novembro de 2020.

ORGANIZAÇÃO MUNDIAL DA SAÚDE - OMS. Painel do WHO Coronavirus Disease (COVID-19). 2020. Disponível em: < https://covid19.who.int/>. Acesso em novembro de 2020.

PEREIRA, M.D. et al. A pandemia de COVID-19, o isolamento social, consequências na saúde mental e estratégias de enfrentamento: uma revisão integrativa. Research, Society and Development, v. 9, n. 7, p. e652974548-e652974548, 2020.

TAVARES, C. A. M. Alterações da ECA2 e Fatores de Risco para Gravidade da COVID-19 em Pacientes com Idade Avançada. Arq Bras Cardiol. v.115, n.4, 2020. Disponível em: $<$ https://www.scielo.br/pdf/abc/v115n4/1678-4170-abc-115-04-070 1.pdf $>$. Acesso em novembro de 2020. 